\title{
Reduced expression of PDCD5 is associated with high-grade astrocytic gliomas
}

\author{
HAIYAN LI ${ }^{1 *}$, QUN WANG ${ }^{1 *}$, FEI GAO ${ }^{1}$, FALIANG ZHU $^{1}$, \\ XIAOYAN WANG ${ }^{1}$, CHENGJUN ZHOU ${ }^{2}$, CHUNMEI LIU ${ }^{1}$, YINGYU CHEN ${ }^{3}$, \\ CHUNHONG MA ${ }^{1}$, WENSHENG SUN ${ }^{1}$ and LINING ZHANG ${ }^{1}$
}

\author{
${ }^{1}$ Institute of Immunology, School of Medicine, Shandong University, Jinan, Shandong 250012; \\ ${ }^{2}$ Department of Pathology, The Second Hospital of Shandong University, Jinan, Shandong 250021; \\ ${ }^{3}$ Center for Human Disease Genomics, Peking University, 38 Xueyuan Road, Beijing 100083, P.R. China
}

Received January 17, 2008; Accepted March 24, 2008

DOI: 10.3892/or_00000044

\begin{abstract}
Programmed cell death 5 (PDCD5) is a novel apoptosis-promoting protein. Recently, a decreased expression of PDCD5 has been found in several types of human tumors. However, the expression level of PDCD5 in human gliomas has not been investigated. In the present study, we examined the expression of PDCD5 in 88 human glioma samples at both mRNA and protein levels by RT-PCR, Western blotting and immunohistochemistry. We found that $53.3 \%$ (16/30) of the glioma samples had a reduced expression of PDCD5 mRNA and $70.5 \%(62 / 88)$ had a reduced expression of the PDCD5 protein as compared to normal brain tissue. Furthermore, we studied the correlation of the expression level of PDCD5 with the clinicopathological grade and survival of patients with astrocytomas. Although longitudinal studies of a cohort of patients with astrocytoma revealed that PDCD5 expression was not able to predict clinical outcome ( $>00.05$ ), a decreased expression of PDCD5 correlated significantly with high-grade astrocytomas $(\mathrm{p}<0.001)$. In conclusion, our data suggest that reduced PDCD5 expression may contribute to the pathogenesis of human gliomas.
\end{abstract}

\section{Introduction}

Programmed cell death 5 (PDCD5), also designated as TF-1 cell apoptosis-related gene-19 (TFAR19), is a strong candidate of apoptosis-regulating proteins because of its known role in programmed cell death (1). Transient or stable overexpression of PDCD5 in TF-1, MGC-803 and HeLa cells enhances

Correspondence to: Dr L. Zhang, Institute of Immunology, School of Medicine, Shandong University, 44\# Wenhuaxi Road, Jinan, Shandong 250012, P.R. China

E-mail: immuno@sdu.edu.cn or zhang_lining@hotmail.com

${ }^{*}$ Contributed equally

Key words: programmed cell death 5, glioma, gene expression programmed cell death triggered by growth factor withdrawal or serum withdrawal from a culture medium (1-3) and increases TAJ/TROY-induced paraptotic cell death (4). Recent studies have shown that the expression of PDCD5 mRNA or protein is down-regulated in certain human tumor tissues, such as gastric cancer (5), lung cancer (6), renal clear cell carcinoma (7), cervical cancer (8), hepatocellular carcinoma (9), acute and chronic myeloid leukemia (10). Additionally, the result from a whole-genome scan of lung cancer demonstrates that patients with genotype GG or GC for SNPs of the 5' region of PDCD5, compared to those with a common genotype CC show an increased risk of lung cancer and a higher incidence of poor clinical stage disease and short-term survival rate, suggesting PDCD5 may represent a novel tumor suppressor gene influencing lung cancer (6). However, PDCD5 expression in other tumors remains unclear.

Human glioma is one of the most frequent primary tumors of the central nervous system. Nearly half of them are malignant and their treatment represents one of the most formidable challenges in clinical medicine. Over the past two decades, the overall survival rate of patients with gliomas has hardly improved and only $\sim 2 \%$ of patients aged 65 years or older survived for $>2$ years $(11,12)$. Therefore, new diagnostic and therapeutic strategies are required in order to control this devastating disease. Recently, considerable progress has been made in understanding the genetic alterations that are associated with gliomas, including oncogenes and tumor suppressor genes $(13,14)$. To date, however, the role of PDCD5 in human glioma has not been investigated.

In this study, the status of PDCD5 expression in 88 primary gliomas was examined by means of RT-PCR, Western blotting and immuohistochemistry. Furthermore, we analyzed the association of PDCD5 expression with clinopathological features and survival of patients.

\section{Materials and methods}

Cell lines and carcinoma specimens. U251 and U87 cell lines derived from gliomas were purchased from the Shanghai Cell Bank of the Chinese Academy of Sciences (Shanghai, P.R. China). They were cultured and maintained in Dulbecco's 
Table I. Correlation between PDCD5 protein expression and clinicopathological features of glioma patients.

\begin{tabular}{|c|c|c|c|}
\hline Clinicopathological variables & PDCD5 (low) & PDCD5 (high) & P-value \\
\hline \multicolumn{4}{|l|}{ Total no. $(n=88)$} \\
\hline \multicolumn{4}{|l|}{ Gender } \\
\hline Male & 41 & 13 & 0.1563 \\
\hline Female & 21 & 13 & \\
\hline \multicolumn{4}{|l|}{ Age (y) } \\
\hline \multicolumn{4}{|l|}{ Median (40) } \\
\hline$>40$ & 35 & 13 & 0.5792 \\
\hline$\leq 40$ & 27 & 13 & \\
\hline \multicolumn{4}{|l|}{ Histological type } \\
\hline Astrocytomas & 57 & 24 & 0.9531 \\
\hline Other type & 5 & 2 & \\
\hline \multicolumn{4}{|l|}{ Grade (Astrocytomas) } \\
\hline I-II (I, I-II, II) & 14 & 17 & $<0.0001$ \\
\hline III-IV (II-III, III, IV) & 43 & 7 & \\
\hline
\end{tabular}

modified Eagle's medium (DMEM) and Modified Eagle's medium (MEM) (Gibco-BRL, Carlsbad, CA, USA) containing $10 \%$ fetal calf serum (Gibco-BRL), respectively. A total of 88 glioma specimens including 30 frozen and 58 paraffinembedded tissues were obtained from patients aged between 30 and 60 years (median 40 years) who underwent operations at the Department of Neurosurgery in Qilu Hospital of Shandong University from October 2003 to March 2006. The pathological diagnosis was made according to the new World Health Organization (WHO) criteria for gliomas. The patient profiles are presented in Table I. None of the patients in this study had received adjuvant immuno-suppressive treatments such as radiotherapy or chemotherapy prior to surgery in order to eliminate their effects on gene expression. Three non-tumorous brain tissue samples were excised from tumor-adjacent sites. This study is in full compliance with the national legislation and the ethical standards of the Chinese Medical Association.

Antibodies and reagents. Mouse anti-human PDCD5 monoclonal antibody is a gift from Dr Dalong Ma (the Center for Human Disease Genomics, Peking University, Beijing, P.R. China). Rabbit anti-human B-actin polyclonal antibody was purchased from Santa Cruz Biotechnology, Inc. (CA, USA). Peroxidase-conjugated goat anti-mouse IgG and Peroxidase-conjugated goat anti-rabbit IgG were purchased from KPL Co. (MD, USA). TRIzol ${ }^{\circledR}$ was purchased from Sangon Biotech Co., Ltd (Shanghai, P.R. China) and the Reverse-Transcribe kit was purchased from Promega Co. (Madison, USA). The PCR MIX was obtained from Tiangen Reagent Co. (Beijing, P.R. China).

RNA isolation and RT-PCR. Total RNAs were extracted from 30 frozen glioma specimens, three frozen normal tissue specimens and two glioma cell lines (U251 and U87) using a modified TRIzol one-step extraction method (Sangon Biotech Co., Ltd) $(15,16)$. cDNA was synthesized using the ReverseTranscribe kit (Promega Co.) according to manufacturer's instructions. The reaction was performed at $70^{\circ} \mathrm{C}$ for $10 \mathrm{~min}$ and then at $42^{\circ} \mathrm{C}$ for $1 \mathrm{~h}$, followed at $95-99^{\circ} \mathrm{C}$ for $5 \mathrm{~min}$ to stop the reaction. PCR was performed using PDCD5 specific primers (sense 5'-CCA TGG CGG ACG AGG AGC TTG-3', and anti-sense 5'-TCA ATA ATC GTC ATC TTC ATC-3') for 30 cycles at $94^{\circ} \mathrm{C}$ for $30 \mathrm{sec}, 58^{\circ} \mathrm{C}$ for $30 \mathrm{sec}$ and $72^{\circ} \mathrm{C}$ for $30 \mathrm{sec}$ followed by an extension cycle at $72^{\circ} \mathrm{C}$ for $7 \mathrm{~min}$.

SDS-PAGE and Western blotting. The proteins were extracted from tissue samples and glioma cell lines using a modified TRIzol one-step extraction method $(15,16)$. The concentration of the protein was determined by Bradford analysis. The protein extract was dissolved in a loading buffer $(1 \mathrm{mM}$ Tris. $\mathrm{Cl}, 3 \%$ SDS, 60\% glycerol and $75 \mathrm{mM}$ DTT) and each sample was analyzed by SDS-PAGE on a $15 \%$ gel. The PVDF membrane was incubated with mouse anti-human PDCD5 (1:1000) or rabbit anti-human 3 -actin (1:2000) at $4^{\circ} \mathrm{C}$ overnight. Immunoreactive bands were visualized using the enhanced chemiluminescence method according to the manufacturer's instructions (ECL, Amersham Biosciences, UK).

Immunohistochemistry. Paraffin sections $(5 \mu \mathrm{m})$ were stained with an anti-PDCD5 antibody by incubating overnight at $4{ }^{\circ} \mathrm{C}$. Secondary staining with biotin-conjugated anti-mouse IgG and tertiary staining with HRP-conjugated strepto-avidin were performed using an $\mathrm{ABC}$ kit and a DAB Peroxidase Substrate kit (Maixin Co., Fuzhou, P.R. China). The sections were counterstained with hematoxylin. Negative controls for the specificity of immunohistochemical reactions were performed by replacing the primary antibody with $\operatorname{IgG}$ of nonimmunized mice.

Scoring of PDCD5 immunohistochemical reaction staining. Cytoplasmic and nuclear expression of PDCD5 was assessed by two independent observers who were blinded to the patient's outcome information. A semi-quantitative scoring system was adopted to evaluate the result of immunohistochemistry and a 
consensus score was reached for each section. The PDCD5 signal was recorded in four grades according to the intensity of the staining: $0,1+, 2+$ and $3+$. The percentages of PDCD5positive cells were also recorded in four categories: $1(0-25 \%)$, $2(26-50 \%), 3(51-75 \%)$ and $4(76-100 \%)(17)$. In the cases with a discrepancy between the two observers, the average score from the two tissue cores was taken as the final score. The sum of the intensity and percentage scores is used as the final staining score. The final staining pattern of the biopsies was defined as follows: 1, negative or faint positive; 2 , weak; 3 , moderate; and 4, strong. Scores 1 and 2 are classified as low expression of PDCD5; whereas 3 and 4 are classified as high expression (overexpression) of PDCD5.

Statistical analysis. The $\chi^{2}$ test was used to compare the expression of PDCD5 with clinicopathological parameters. Cumulative survival time was calculated by the Kaplan-Meier method and analyzed by the log-rank test. $\mathrm{P}<0.05$ was considered statistically significant. The calculations were performed using the SPSS statistical software.

\section{Results}

Expression of PDCD5 in human glioma-derived cell lines. To explore the potential roles of PDCD5 in gliomas, the status of PDCD5 expression in glioma-derived cell lines, U251 and U87, was examined by RT-PCR and Western blotting. As shown in Fig. 1, the U251 cell line expressed high levels of
A

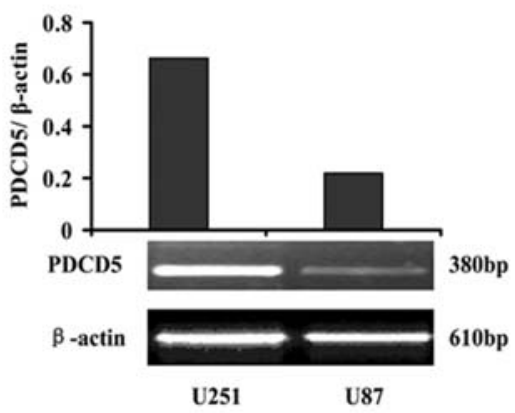

B

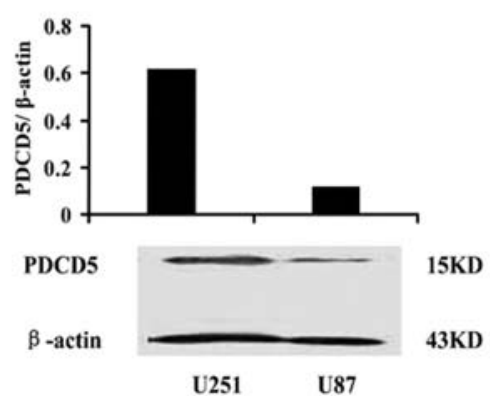

Figure 1. Expression of PDCD5 mRNA and protein in human glioma-derived cell lines. (A) The expression of PDCD5 mRNA in glioma-derived U251 and U87 cell lines was detected by RT-PCR. B-actin was used as the internal loading control. The upper panel shows the normalized PDCD5 expression level. (B) The expression level of the PDCD5 protein was detected by Western blotting. The upper panel shows the normalized PDCD5 expression level.
A
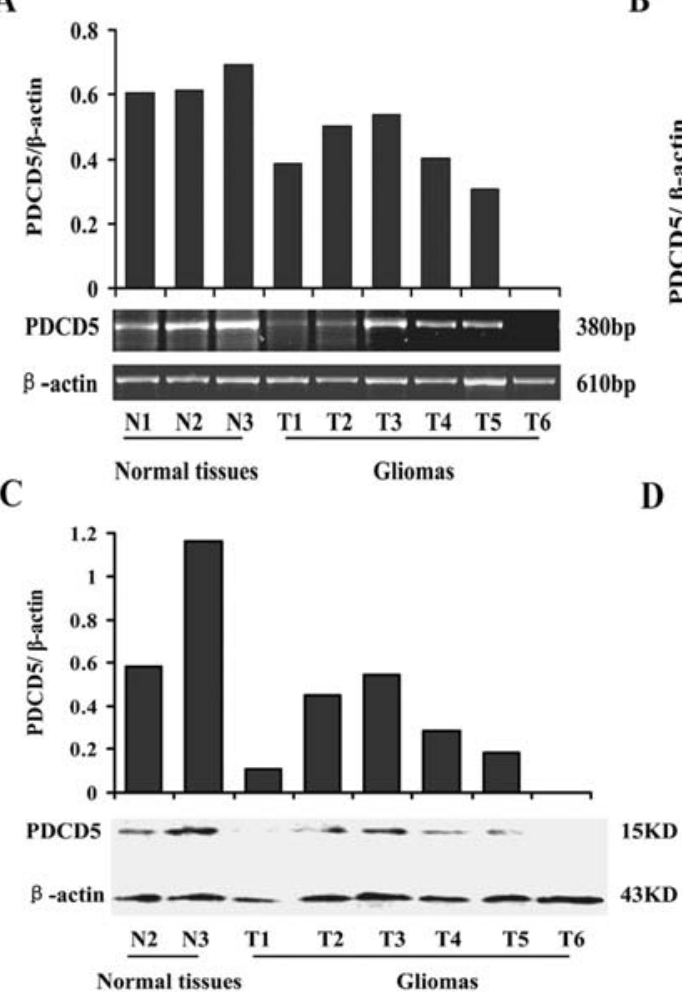

D
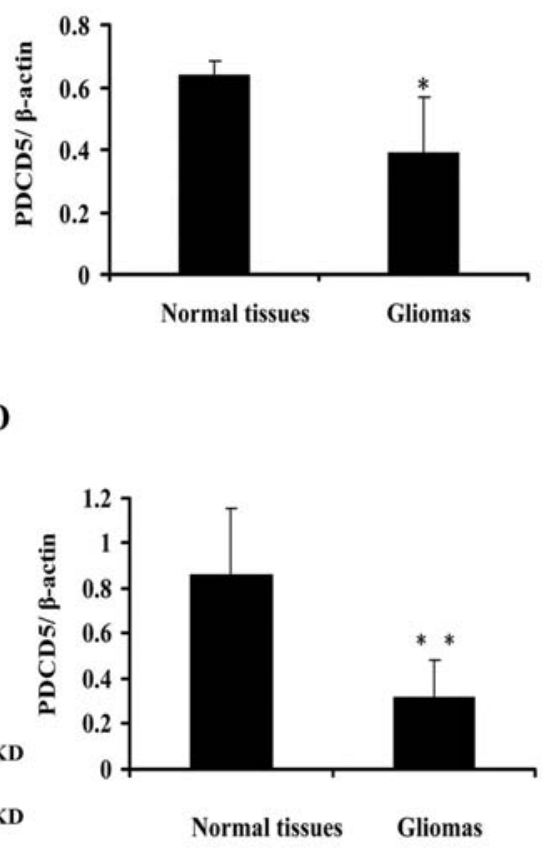

Figure 2. Expression of PDCD5 mRNA and protein in human primary glioma samples. (A) The expression levels of PDCD5 mRNA and protein in human non-tumorous brain tissues (N1-3) and in six representative primary glioma samples (T1-T6) were detected by RT-PCR and the result was normalized with B-actin. (B) Normalized PDCD5 mRNA levels in 30 frozen glioma samples and three non-tumorous brain tissues were analyzed. The difference is statistically significant $\left({ }^{*} \mathrm{p}<0.05\right)$. (C) The expression levels of the PDCD5 protein in two human non-tumorous brain tissue (N2-3) and in six representative primary gliomas samples (T1-T6) was detected by Western blotting. (D) Normalized PDCD5 protein levels in 30 frozen glioma samples and three normal brain tissues were analyzed. The difference is statistically significant $\left({ }^{* *} \mathrm{p}<0.01\right)$. 
Table II. Analysis of PDCD5 expression in gliomas

\begin{tabular}{|c|c|c|c|c|}
\hline No. & RNA & Protein (Western blot) & Grade & Classification \\
\hline N1 & +++ & +++ & & \\
\hline $\mathrm{N} 2$ & +++ & ++++ & & \\
\hline N3 & +++ & ++++ & & \\
\hline 1 & ++ & + & GIV & Astrocytomas \\
\hline 2 & +++ & ++ & GIV & Astrocytomas \\
\hline 3 & +++ & +++ & GII-III & Anaplastic oligodendroglioma \\
\hline 4 & +++ & ++ & GI-II & Astrocytomas \\
\hline 5 & ++ & + & GI-II & Central neurocytoma \\
\hline 6 & - & - & GIV & Astrocytomas \\
\hline 7 & ++ & ++ & GIV & Astrocytomas \\
\hline 8 & ++ & + & GIV & Astrocytomas \\
\hline 9 & ++ & + & GIV & Medulloblastoma \\
\hline 10 & +++ & +++ & GIV & Astrocytomas \\
\hline 11 & +++ & + & GII-III & Astrocytomas \\
\hline 12 & +++ & + & GII-III & Astrocytomas \\
\hline 13 & + & +++ & GII-III & Anaplastic oligodendroglioma \\
\hline 14 & ++ & ++ & GII-III & Oligodendroglioma \\
\hline 15 & +++ & ++ & GIV & Astrocytomas \\
\hline 16 & +++ & ++ & GII-III & Astrocytomas \\
\hline 17 & + & ++ & GIV & Astrocytomas \\
\hline 18 & + & ++ & GIV & Astrocytomas \\
\hline 19 & ++ & + & GII-III & Astrocytomas \\
\hline 20 & + & + & GIV & Anaplastic oligodendroglioma \\
\hline 21 & +++ & ++ & GIV & Astrocytomas \\
\hline 22 & +++ & + & GII-III & Astrocytomas \\
\hline 23 & +++ & ++ & GIV & Astrocytomas \\
\hline 24 & +++ & ++ & GII-III & Anaplastic oligodendroglioma \\
\hline 25 & ++ & + & GII-III & Astrocytomas \\
\hline 26 & + & ++ & GII-III & Astrocytomas \\
\hline 27 & ++ & + & GIV & Astrocytomas \\
\hline 28 & +++ & +++ & GII-III & Astrocytomas \\
\hline 29 & +++ & ++ & GIV & Astrocytomas \\
\hline 30 & ++ & +++ & GII-III & Astrocytomas \\
\hline
\end{tabular}

The pathological analysis was performed according to the new World Health Organization (WHO) criteria. The levels of PDCD5 mRNA were analyzed by semi-quantitative RT-PCR according to OD values of PDCD5 mRNA expression normalized to $\beta$-actin: -, not detected; +, 0 to 0.25 ; ,++ 0.25 to 0.5 and,$+++>0.5$. Expression of the PDCD5 protein was detected by Western blotting semi-quantitatively according to OD values of PDCD5 protein expression normalized to $\beta$-actin: -, not detected;,+ 0 to $0.25 ;++, 0.25$ to $0.5 ;+++, 0.5$ to 0.75 and,$++++>0.75$.

PDCD5 mRNA and protein, whereas the U87 cell line showed low levels of PDCD5 expression, suggesting that the expression of PDCD5 may be reduced in human gliomas.

Expression of PDCD5 in human primary gliomas. Then, the expression of PDCD5 mRNA and protein in human primary gliomas was examined by RT-PCR and Western blotting. Compared to three normal brain samples, $53.3 \%$ (16/30) of the human primary glioma samples expressed low or no PDCD5 mRNA (Fig. 2A and B) (Table II), while $83.3 \%$ $(25 / 30)$ of them showed a reduced expression of the PDCD5 protein (Fig. 2C and D) (Table II).

Additionally, we examined PDCD5 expression by immunohistochemisty. As shown in Fig. 3A, PDCD5 was predominantly expressed in the cytoplasm, although a low level of PDCD5 was also detected in the nucleus, as previously reported (18). In non-tumorous brain tissues, there was a strong positive staining of PDCD5 (Fig. 3A-a). In grade I-II of the glioma samples, there was a moderate positive staining of PDCD5 (Fig. 3A-b). In contrast, in grade III of the glioma samples, there was a weak positive staining of PDCD5 (Fig. 3A-c). In grade IV of the glioma samples, faint or no staining of PDCD5 was detected in the tumor cells (Fig. 3A-d). The positive rate and intensity of PDCD5 expression were further analyzed as described in Materials and methods. A correlation of the expression levels of PDCD5 with clinicopathological grades of gliomas is shown in Fig $3 \mathrm{~B}$. Glioma samples (70.5\%) (62/88) showed low or no expression 
A

$\mathbf{a}$

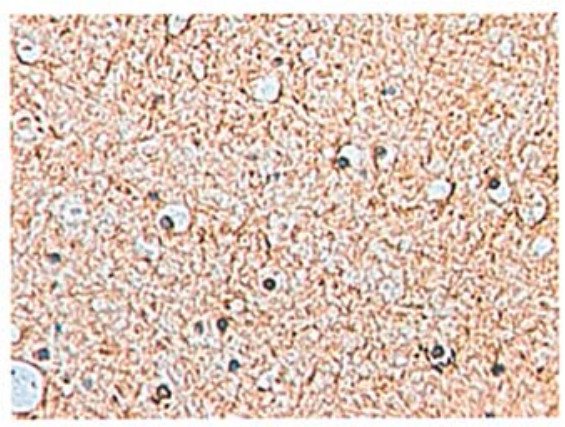

c

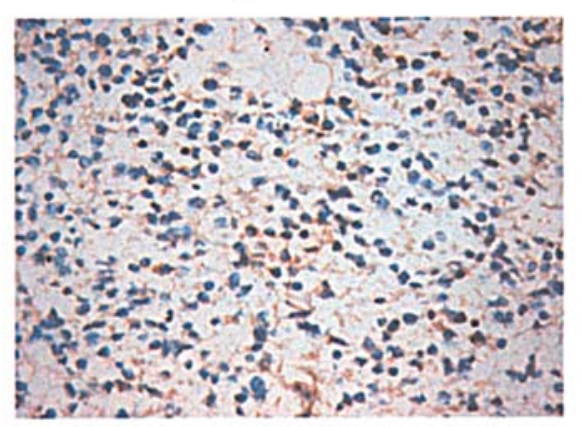

B

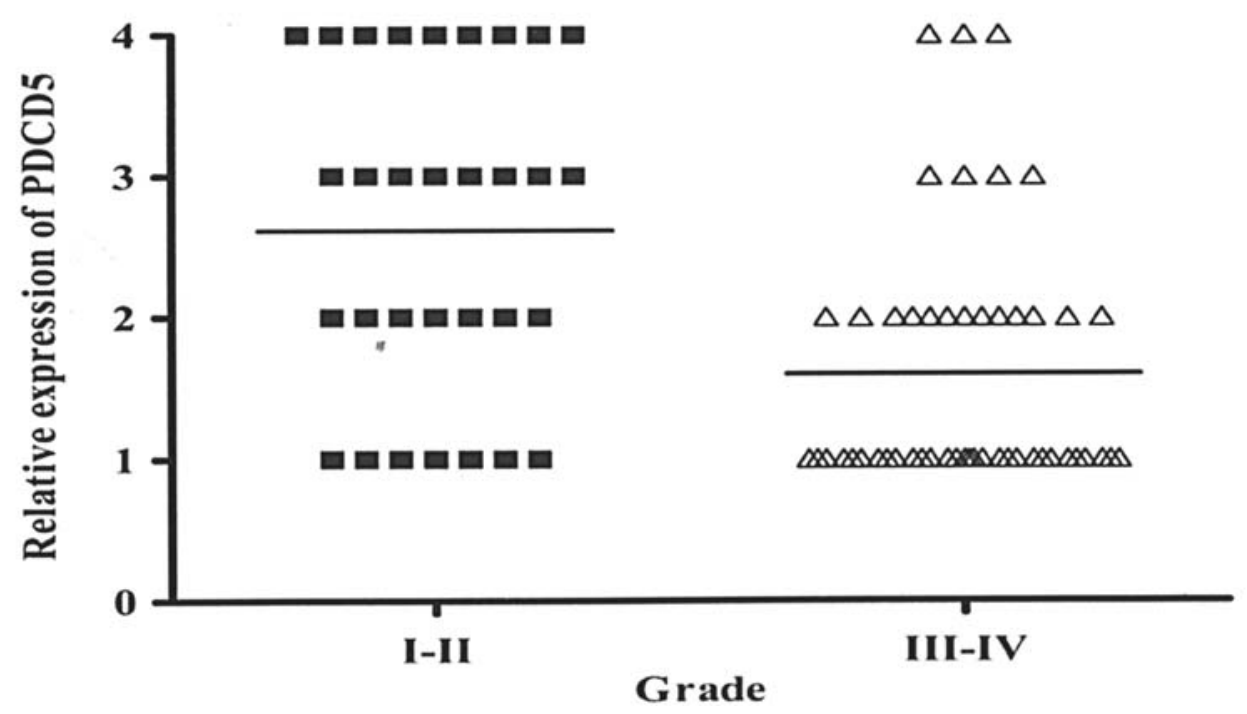

b

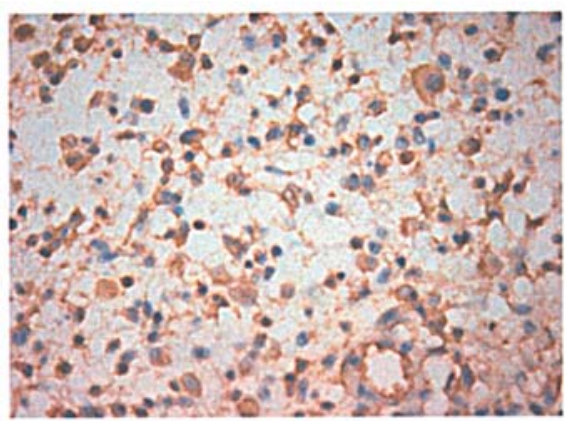

d

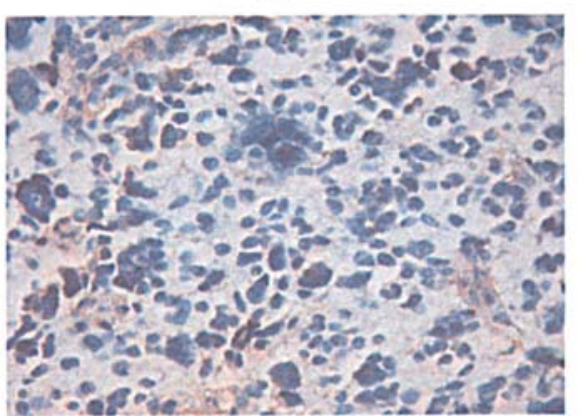

Figure 3. Representative images of PDCD5 immunohistochemical staining in normal brain and glioma tissues. (A) Normal brain tissue samples (a) showed strong staining in the nucleus and cytoplasm. In contrast, glioma tissues (b-d) had weak or reduced specific staining. Original magnifications, $\mathrm{x} 400$. (B) Distribution of PDCD5 staining intensity in glioma samples. According to the grade of gliomas, the samples were divided into two groups, low grade (I-II) and high grade (III-IV). The staining intensity of the anti PDCD5 antibody was scored as 1-4 as described in Materials and methods.

of PDCD5 (Table I). These results indicate that the expression of PDCD5 was frequently reduced in human primary gliomas.

Correlation of the expression level of PDCD5 with the clinicopathological grade and survival of patients with primary gliomas. To determine the clinical value of PDCD5 expression in primary gliomas, we examined the correlation of PDCD5 expression with the clinicopathological grade and survival of patients with gliomas. The results showed that there was no significant correlation between PDCD5 expression and gender, age or histological type (Table I). However, the expression of PDCD5 correlated significantly with the pathological grade $(\mathrm{p}<0.001)$. Within the same histological glioma (astrocytoma) type, the rate of reduced expression of PDCD5 was higher in high-grade gliomas (III-IV) than that in low-grade gliomas (I-II) $(86.0 \%, 43 / 50$ vs. $45.2 \%, 14 / 31)$ (Table I). Furthermore, we analyzed the association of the overall survival of the astrocytoma patients $(n=81)$ with low or high PDCD5 expression. However, as judged by the Kaplan-Meier analysis (Fig. 4A), PDCD5 expression had no statistically significant impact on the prognosis of astrocytoma patients $(\mathrm{p}>0.1)$. Similarly, no progonostic value of PDCD5 expression can be detected for patients with same grades of gliomas (I-II, n=31; III-IV, n=50) (Fig. 4B and C). 
$\mathbf{A}$

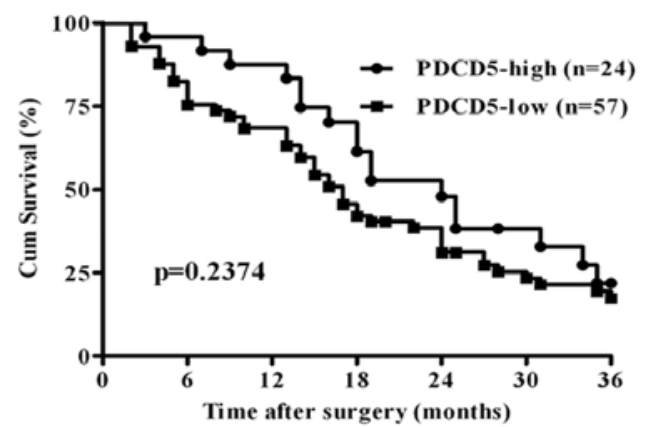

B

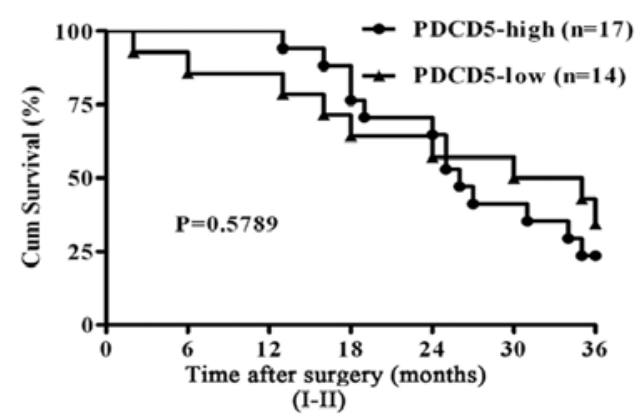

C

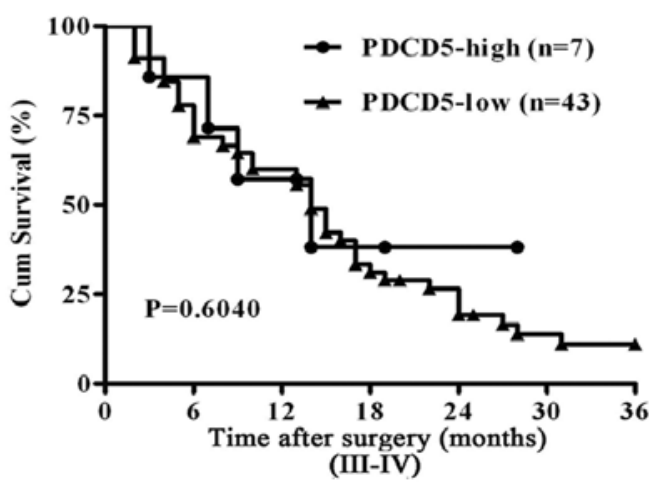

Figure 4. Correlation between the expression level of the PDCD5 protein and the survival of patients with astrocytomas. (A) The prognostic value (patient outcome) of PDCD5 in 81 astrocytoma patients with complete data was analyzed by Kaplan-Meier survival analysis and the log-rank test $(\mathrm{p}=0.2374)$. (B) The prognostic value of PDCD5 in 31 low-grade (I-II) astrocytoma patients was analyzed $(\mathrm{p}=0.5789)$. (C) The prognostic value of PDCD5 in 50 high-grade (III-IV) astrocytoma patients was analyzed $(\mathrm{p}=0.6040)$.

\section{Discussion}

In the present study, we demonstrated for the first time, that PDCD5 expression decreased significantly in gliomas compared with non-tumorous brain tissues, which significantly correlated with the grades of the gliomas. However, longitudinal studies of a cohort of patients revealed that PDCD5 expression had no statistically significant impact on the prognosis of astrocytoma patients.

The expression of PDCD5 increases during the apoptotic process of cells induced by cytokine withdrawal (1). The overexpression of PDCD5 promotes programmed cell death triggered by certain stimuli $(2,3)$. Importantly, blocking the activity of PDCD5 by introducing an anti-PDCD5 antibody into cells is able to suppress the etoposide-induced apoptosis of tumor cells (3). These reports demonstrate that PDCD5 plays a significant role in apoptotic and paraptotic cell deaths. It is well known that the dysfunction of cell apoptosis leads to uncontrolled cell growth, such as cancer $(19,20)$. In the present study, we demonstrated that the expression of PDCD5 mRNA or protein decreased significantly in primary gliomas, compared with normal brain tissues (Figs. 2 and 3) (Table II), which is consistent with other reports of cervical cancer (8), gastric tumor (5), lung cancer (6) and chronic myelogenous leukemia (10). Thus, the results suggest that a low expression of PDCD5 in tumors may affect the growth of tumors including glioma by interfering with programmed cell death. However, more studies are needed to clarify this issue.

To date, the clinical significance of PDCD5 downregulation in tumors is unclear. Previous reports showed that the decreased expression of PDCD5 in renal clear cell carcinomas significantly correlated with the high-grade tumor (7) and that the patients with low PDCD5-expressing gastric tumor have a shorter survival period (5). Astrocytic tumors, the most common type of gliomas, develop from a type of star-shaped cell called astrocytes. To clarify the clinical significance of PDCD5 down-regulation in gliomas, we divided astrocytic tumors into two groups: low-grade group (grades I and II ) and high-grade group (grade III and IV) according to the World Health Organization (WHO) classification system and then analyzed the association of PDCD5 expression in two groups of astrocytomas with clinicopathological characteristics and survival of patients by a three-year follow-up investigation. Our results demonstrated that the reduced expression of PDCD5 correlated significantly with high-grade astrocytoma. In general, low-grade astrocytomas are usually slow growing and are not likely to spread, whereas high-grade astrocytomas grow rapidly and have a poor prognosis. However, longitudinal studies of a cohort of patients revealed that the status of PDCD5 expression has no significant correlation with the prognosis of patients. The lack of correlation is most likely explained by the multiple prognostic factors involved in the disease, such as diagnosis or surgery time and the complex network of other genes, which are involved in the tumor formation. However, the lack of prognostic impact in our study population does not exclude a potential role for PDCD5 in the pathogenesis of human gliomas.

In conclusion, we have demonstrated that a decreased expression of PDCD5 correlated significantly with highgrade astrocytomas. Although the expression of PDCD5 did not influence clinical outcome in our study, PDCD5 may serve as a novel therapeutic target for the treatment of cancer.

\section{Acknowledgements}

We particularly thank Dr Dalong Ma from the Center for Human Disease Genomics, Peking University, P.R. China for kindly presenting us with the anti-PDCD5 antibody. We are grateful to Dr Changjun Zhu from the Institute of cell biology, School of Medicine, Shandong University, P.R. China for his valuable suggestions and kind help. This study was supported by grants from the Natural Science Foundation of China (No. 30628015) and the National '973' program (No. 2006CB503803). 


\section{References}

1. Liu HT, Wang YG, Zhang YM, et al: TFAR19, a novel apoptosis-related gene cloned from human leukemia cell line TF-1, could enhance apoptosis of some tumor cells induced by growth factor withdrawal. Biochem Biophys Res Commun 254: 203-210, 1999.

2. Zhang YM, Xu XZ, Liu HT, Song QS and Ma DL: The apoptosis-accelerating effect of human recombinant TFAR19 protein on leukemia HL-60 cells. J Chin Immunol 16: 8-11, 2000.

3. Rui M, Chen YY, Zhang YM and Ma DL: Transfer of antiTFAR 19 monoclonal antibody into HeLa cells by in situ electroporation can inhibit the apoptosis. Life Sci 71: 1771-1778, 2002.

4. Wang Y, Li XT, Wang L, Ding PG, Zhang YM, Han WL and Ma DL: An alternative form of paraptosis-like cell death, triggered by TAJ/TROY and enhanced by PDCD5 overexpression. J Cell Sci 117: 1525-1532, 2004.

5. Yang YH, Zhao M, Li WM, Chen YY, Kang B and Lu YY: Expression of programmed cell death 5 gene involves in regulation of apoptosis in gastric tumor cells. Apoptosis 11: 993-1001, 2006.

6. Spinola M, Meyer P, Kammerer S, et al: Association of the PDCD5 locus with lung cancer risk and prognosis in smokers. J Clin Oncol 24: 1672-1678, 2006.

7. Tan WL, Xiong L, Zheng SB, Yu ZC, Qi H, Du YJ and Wu P: Relationship between programmed cell death 5 protein expression and prognosis of renal clear cell carcinoma. Nan Fang Yi Ke Da Xue Xue Bao 26: 1316-1318, 2006.

8. Liu ZH, Zhang D, Li KM and Liao QP: Expression of PDCD5 in tissues of normal cervix, CIN I-III and cervical cancer. Beijing Da Xue Xue Bao 36: 407-410, 2004.

9. Xu XR, Huang J, Xu ZG, et al: Insight into hepatocellular carcinogenesis at transcriptome level by comparing gene expression profiles of hepatocellular carcinoma with those of corresponding noncancerous liver. Proc Natl Acad Sci USA 98: 15089-15094, 2001.
10. Ruan GR, Qin YZ, Chen SS, et al: Abnormal expression of the programmed cell death 5 gene in acute and chronic myeloid leukemia. Leukemia Res 30: 1159-1165, 2006.

11. Bansal K, Liang ML and Rutka JT: Molecular biology of human gliomas. Technol Cancer Res Treat 5: 185-194, 2006.

12. Nakabayashi $H$, Hara $M$ and Shimuzu K: Clinicopathologic significance of cystatin C expression in gliomas. Hum Pathol 36: $1008-1015,2005$

13. Perunovic B, Athanasiou A, Quilty RD, Gorgoulis VG, Kittas C and Love S: Expression of mos in astrocytic tumors and its potential role in neoplastic progression. Hum Pathol 33: 703-707, 2002.

14. Gao F, Zhang P, Zhou C, Li J, Wang Q, Zhu F, Ma C, Sun W and Zhang L: Frequent loss of PDCD4 expression in human glioma: possible role in the tumorigenesis of glioma. Oncol Rep 17: 123-128, 2007.

15. Chadderton T, Wilson C, Bewick M and Gluck S: Evaluation of three rapid RNA extraction reagents: relevance for use in RTPCR's and measurement of low level gene expression in clinical samples. Cell Mol Biol 43: 1227-1234, 1997.

16. Culley DE, Kovacik WP Jr, Brockman FJ and Zhang W: Optimization of RNA isolation from the archaebacterium Methanosarcina barkeri and validation for oligonucleotide microarray analysis. J Microbiol Methods 67: 36-43, 2006.

17. Dai DL, Martinka M and Li G: Prognostic significance of activated Akt expression in melanoma: a clinico-pathologic study of 292 cases. J Clin Oncol 23: 1473-1482, 2005.

18. Sun CL, Qiao J, Hu ZX, Zhang T and Chen YY: Expression of novel apoptosis-related protein PDCD5 in granulosa cells of polysystic ovary syndrome. Beijing Da Xue Xue Bao 37: 476-479, 2005.

19. McCabe ML and Dlamini Z: The molecular mechanisms of oesophageal cancer. Int Immunopharmacol 5: 1113-1130, 2005.

20. Kountouras J, Zavos C and Chatzopoulos D: Apoptotic and antiangiogenic strategies in liver and gastrointestinal malignancies. J Surg Oncol 90: 249-259, 2005. 